A

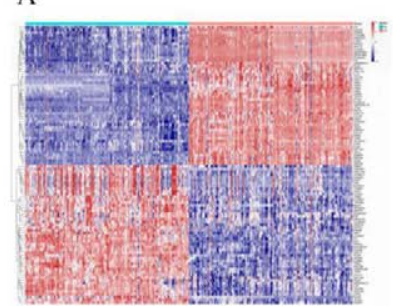

$\mathrm{C}$

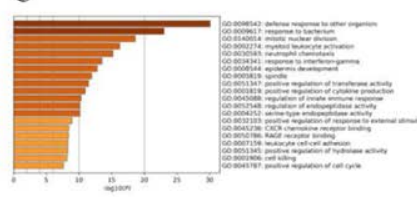

E

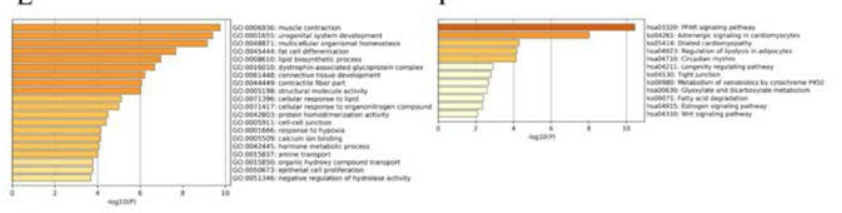

G

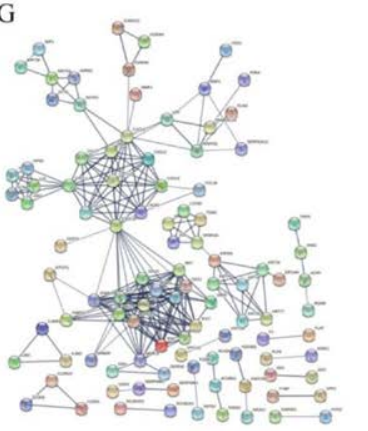

B

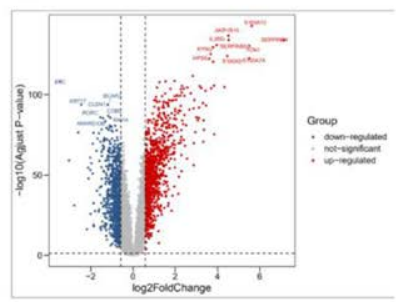

$\mathrm{D}$

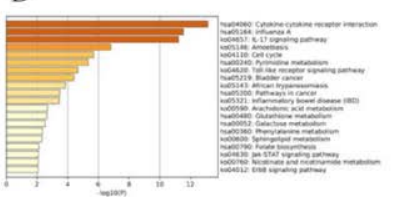

$\mathrm{F}$

$\mathrm{H}$

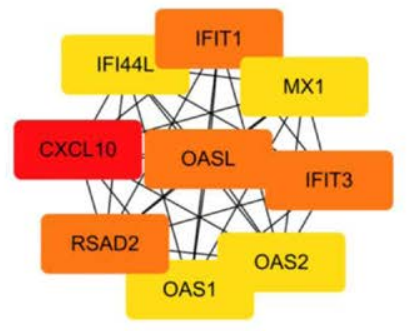

Figure 1: (A)Heatmap of DEGs: The red and blue colors represent up-regulation and downregulation, respectively (B) Volcano map of gene expression: The horizontal axis is $\log 2 \mathrm{FC}$, the vertical axis is $\log 10$ adj.PVal. Each dot represents a gene, blue indicates low expression genes, (G,H)PPl network were screened by Cytoscape plugin Cytohubba based on their degree value (high degree value) to yellow (low degree value).

Acknowledgements: This project was supported by National Science Foundation of China (82001740), Open Fund from the Key Laboratory of Cellular Physiology (Shanxi Medical University) (KLCP2019) and Innovation Plan for Postgraduate Education in Shanxi Province (2020BY078).

Disclosure of Interests: None declared

DOI: 10.1136/annrheumdis-2021-eular.1773

\title{
AB0006 GENETIC RISK PROFILE FOR PSORIATIC ARTHRITIS PREDISPOSITION IN ITALIAN PATIENTS
}

M. S. Chimenti ${ }^{1}$, C. Ciccacci ${ }^{2}$, G. De Benedittis ${ }^{3}$, A. Latini ${ }^{3}$, P. Conigliaro ${ }^{1}$, G. L. Fonti ${ }^{1}$, P. Triggianese ${ }^{1}$, P. Borgiani ${ }^{3}$, R. Perricone ${ }^{1} .{ }^{1}$ University of Rome Tor Vergata, Rheumatology, Allergology and Clinical Immunology, Rome, Italy; ${ }^{2}$ UniCamillus - Saint Camillus International University of Health Sciences, Genetics Unit, Rome, Italy; ${ }^{3}$ University of Rome Tor Vergata, Genetics Unit, Rome, Italy

Background: Psoriatic arthritis (PsA) is a chronic inflammatory joint disease typically associated with psoriasis and classified in the group of spondyloarthritis (1). The pathogenesis is based on an interplay of different genes interacting with several environmental factors including stress, trauma, infections, triggering an inflammatory response related to the activation of innate and acquired immunity in different tissues and organs (2). However, the risk for the development of PsA is not clearly understood.

Objectives: The aim of this study was to evaluate, in a cohort of Italian PsA out-patients of the Rheumatology Unit of the University of Rome Tor Vergata, the association of genetic variants in candidate genes for PSA susceptibility and their possible contribute in the modulation of clinical and laboratory features. Methods: The genes were selected according to previous studies describing these genes as involved in susceptibility to rheumatoid arthritis (RA) (3), since

a common genetic background can be shared between these diseases. Nine SNPs (single nucleotide polymorphism) in eight candidate genes were analysed: STAT4 (rs7574865), TRAF3IP2 (rs33980500), TNFAIP3 (rs6920220 and rs2230926), MIR146A (rs2910164), PSORS1C1 (rs2233945), IL-10 (rs1800872), HCP5 (rs3099844) and ERAP1 (rs27524). Polymorphisms were analysed in 163 consecutive PsA out-patients and 198 healthy controls (HC). Genotyping was performed by allelic discrimination by TaqMan assay. Alleles frequencies differences between cases and controls or between phenotypic groups were compared using Pearson's $\chi 2$ test.

Results: We have observed an association between PSA susceptibility and the variant alleles of STAT4 [OR= $1.60(1.15-2.21), \mathrm{P}=0.005]$, TRAF3IP2 [OR= 1.65 (1.01-2.65), $P=0.04]$, ERAP1 [OR= $1.40(1.05-1.85), P=0.02]$ and TNFAIP3 (rs6920220) $[\mathrm{OR}=1.75(1.19-2.57), \mathrm{P}=0.004]$. On the contrary, the variant allele of IL-10 polymorphism seems to play a protective role [OR= $0.74(1.05-1.85), P=$ 0.05]. Moreover, in order to define a genetic risk profile, we have counted the total number of risk alleles in each subject, considering as risk alleles the allelic variant of rs7574865 (STAT4), rs33980500 (TRAF3IP2), rs6920220 (TNFAIP3) and rs27524 (ERAP1) SNPs. Then, we have compared the risk allele number distribution between patients and $\mathrm{HC}$ (Fig.1). Classes with 3 or more risk alleles are significantly more represented in patients than in $\mathrm{HC}(\mathrm{OR}=2.03, \mathrm{P}=0.004)$. The risk to develop the disease increases significantly in subjects with at least four risk alleles $(\mathrm{OR}=2.96, \mathrm{P}=0.002)$.

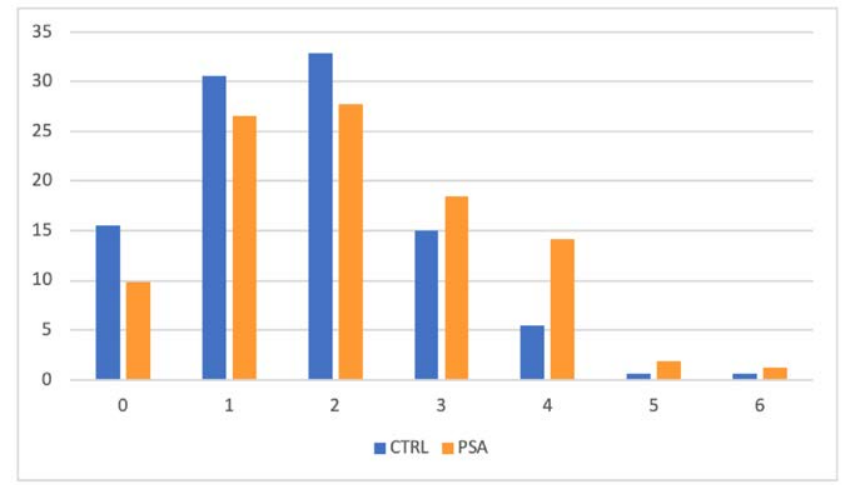

Figure 1. Number of risk alleles in patients and controls: rs7574865 (STAT4), rs33980500 (TRAF3IP2), rs6920220 (TNFAIP3) and rs27524 (ERAP1) SNPs.

Conclusion: We confirm the associations between five SNPs, already studied in RA, and PSA susceptibility, suggesting a common inflammatory pathway in chronic inflammatory rheumatological diseases. Moreover, we show how the genotyping of only few associated SNPs could help to define a genetic risk profile for PSA development.

\section{REFERENCES:}

[1] Calabresi E, et al. One year in review 2019: psoriatic arthritis. Clin Exp Rheumatol. 2020;38:1046-55.

[2] Chimenti MS, Triggianese P, De Martino E, Conigliaro P, Fonti GL, Sunzini F, Caso F, Perricone C, Costa L, Perricone R. An update on pathogenesis of psoriatic arthritis and potential therapeutic targets. Expert Rev Clin Immunol. 2019 Aug;15(8):823-836.

[3] Ciccacci C, et al. Polymorphisms in STAT-4, IL-10, PSORS1C1, PTPN2 and MIR146A genes are associated differently with prognostic factors in Italian patients affected by rheumatoid arthritis. Clin Exp Immunol. 2016;186:157-63.

Disclosure of Interests: None declared

DOI: 10.1136/annrheumdis-2021-eular.1976

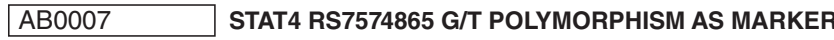 OF PREDISPOSITION TO SYSTEMIC LUPUS ERYTHEMATOSUS WITH JUVENILE ONSET}

M. Kaleda', M. Krylov², I. Nikishina ${ }^{1}$. ${ }^{1}$ V. A. Nasonova Research Institute of Rheumatology, Pediatric Department, Moscow, Russian Federation; ${ }^{2} \mathrm{~V}$. A. Nasonova Research Institute of Rheumatology, Genetic Department, Moscow, Russian Federation

Background: Systemic lupus erythematosus (SLE) is a chronic autoimmune disease with a significant genetic predisposition. Recent studies have identified STAT4 (signal transducers and transcription activators 4) as a susceptibility gene for SLE.

Objectives: To investigate the hypothesis of the association of STAT4 rs7574865 $\mathrm{G} / \mathrm{T}$ polymorphism with the predisposition to SLE in children and its relationship with some of SLE manifestations. 